\title{
ArcheoSciences
}

Revue d'archéométrie

\section{Approche climatique de la période romaine dans l'est du Var : recherche et analyse des composantes périodiques sur un concrétionnement centennal ( $\mathrm{I}^{\mathrm{er}}-\mathrm{II}{ }^{\mathrm{e}}$ siècle apr. J.-C.) de l'aqueduc de Fréjus}

Climatic approach of the roman period in eastern Var : research and analysis of periodic components of a centennial concrétion of the Fréjus aqueduct (1-2 nd $A D)$

\section{Michel Dubar}

\section{OpenEdition}

\section{Journals}

\section{Édition électronique}

URL : https://journals.openedition.org/archeosciences/359

DOI : 10.4000/archeosciences.359

ISBN : 978-2-7535-1595-6

ISSN : 2104-3728

Éditeur

Presses universitaires de Rennes

Édition imprimée

Date de publication : 31 décembre 2006

Pagination : 163-171

ISBN : 978-2-7535-0456-1

ISSN : 1960-1360

Référence électronique

Michel Dubar, «Approche climatique de la période romaine dans l'est du Var : recherche et analyse des composantes périodiques sur un concrétionnement centennal (|er-||e siècle apr. J.-C.) de l'aqueduc de Fréjus », ArcheoSciences [En ligne], 30 | 2006, document 16, mis en ligne le 31 décembre 2008, consulté le 01 février 2022. URL : http://journals.openedition.org/archeosciences/359; DOI : https:// doi.org/10.4000/archeosciences.359 


\title{
Approche climatique de la période romaine dans l'est du Var : recherche et analyse des composantes périodiques sur un concrétionnement centennal $\left(\mathrm{I}^{\mathrm{er}}-\mathrm{II}^{\mathrm{e}}\right.$ siècle apr. J.-C.) de l'aqueduc de Fréjus
}

\author{
Michel Dubar*
}

\begin{abstract}
Résumé : Le concrétionnement de l'aqueduc antique de Fréjus est constitué de 102 lamines carbonatées saisonnières biannuelles. Par référence au régime hydrologique actuel des sources qui l'alimentaient, il apparaît que le concrétionnement est saisonnier et biannuel. Il a fait l'objet d'un décompte et d'une analyse spectrale dans le but de décrypter d'éventuels microcycles climatiques. Les différents spectrogrammes obtenus font essentiellement apparaître un pic robuste de haute fréquence à 7,1-7,3 ans et un pic basse fréquence à 39-43 ans. Cette bipartition des fréquences caractérise l'Oscillation Nord Atlantique (ONA) dont le rôle est prépondérant en Europe occidentale. La haute fréquence correspond au mode actif, la basse fréquence au mode passif. Comme les deux périodicités sont présentes dans la série étudiée, nous pouvons dire que le mode de l'oscillation n'est pas strictement déterminé. L'intervalle considéré, validé à 102 ans par l'analyse spectrale, qui s'étendrait d'après les données archéologiques du milieu du I ${ }^{\text {er }}$ siècle au milieu du II $^{\mathrm{e}}$ siècle, semble présenter une alternance de phases pluridécennales à ONA actif avec des phases à ONA moins actif. L'ambiance climatique estimée par rapport au climat régional actuel peut être qualifiée de tempérée-humide. Ce résultat est cohérent avec les reconstitutions paléoenvironnementales proposées par différents auteurs ces dernières années : la période romaine est, en Provence, caractérisée par un calme érosif, sans cachet climatique bien tranché.
\end{abstract}

Abstract: The travertine concretion of the ancient aqueduct of Frejus consists of 102 doublets of biannual laminae. The present hydrological mode of the sources indicates that the lamination is seasonal and biannual. With the aim of deciphering possible climatic microcycle, it was the subject of a counting and a spectral analysis

The various spectrograms obtained revealed a high frequency robust peak at 7.1-7.3 years and a low frequency peak at 39-43 years. Such bipartition of frequencies characterizes the NAO which dominated the climate of Western Europe and of the Alps. Since the two periods were present in the studied series, it is possible to affirm that the mode of oscillation is not strictly determined. When using a Gaussian filter at 7.3 years, as was to be expected, a low frequency modulation was highlighted at 39-43 years. The considered period of 102 years which extended on the basis of the archaeological data from the mid-Ist century to the mid-IInd century, seems to offer alternative phases of active ONA with phases of less active ONA. It may be described as moderately wet with alternative dryer-colder phases.

This result is consistent with palaeoenvironmental reconstitutions suggested by various authors these last years : the Roman period is, in Provence, characterized by low erosion and without strong climatic feature.

Mots clés : Climat, Antiquité, Provence orientale, Fréjus, aqueduc, concrétionnement, analyse spectrale.

Key words: Climate, Antiquity, Eastern Provence, Fréjus, aqueduct, spectral analysis.

*CNRS, Cépam (UMR 6130), 250 rue Albert-Einstein, 06560 Valbonne. (dubar@cépam.cnrs.fr) 


\section{BUT DE L'ÉTUDE, INTRODUCTION}

On se propose de retrouver dans le concrétionnement travertineux à lamines de l'aqueduc de Fréjus d'éventuels signaux climatiques, plus précisément des micropériodicités susceptibles d'être interprétées en terme de climat par rapport à ce qui est connu dans l'actuel.

La recherche de microcycles d'obédience terrestre (atmosphère, stratosphère, océan) ou solaire intervenant à divers degrés dans les mécaniques du climat est basée sur l'analyse spectrale. Cette méthode a pour objet les séries chronologiques mesurées relatives aux dernières décennies (température, pluviométrie, débits des rivières, régime des vents, etc.). Cette approche a pour but de caractériser au point de vue dynamique des scénarios climatiques du passé proche et de les confronter aux modèles météorologiques en vigueur. Récemment, ce type d'étude a été étendu à des séries remontant dans le temps de quelques siècles : chroniques historiques (Plaut et al., 1995) ou données géologiques lacustres (Chapron et al., 2002) ou karstiques (Burns et al., 2002).

\section{MéThode eT PRINCIPE D’ÉTUde}

Dans le domaine de la géologie, la donnée mesurée ne peut au mieux qu'approcher un paramètre climatique (proxy-mesure). On utilise des mesures de paramètres physiques (indices cristallographiques, susceptibilité magnétique), chimiques (teneur en carbonates, isotopes stables) ou biologiques (pollens ou autres). Pour l'analyse spectrale ces mesures doivent constituer une série chronologique discrète à pas de temps constant.

Dans le domaine de l'étude des concrétionnements carbonatés on utilise la variation binaire classique lamine claire/ lamine sombre qui est en relation avec la cristallographie de la calcite, elle-même reflet des conditions physico-chimiques environnementales : lamine claire à croissance optimale en saison de grande percolation et lamine sombre à petits cristaux, irrégulière, souvent multiple, de la saison sèche. Pour chaque catégorie de lamine, la croissance de l'épaisseur des lamines est fonction du taux de percolation et/ou de la durée de la saison en question (Genty et Quinif, 1996). Il suffira donc, en première analyse, de mesurer l'épaisseur des lamines. Cette composition binaire simple a pour intérêt d'être représentative du régime hydrologique de la cavité ou de la source et de pouvoir être décomptée comme des varves ou comme des cernes de bois. Evidemment pour chaque site il convient de s'assurer, en particulier en s'aidant de l'hydrologie, de l'annuité du doublet (Baker et Genty, 2003). En effet des systèmes laminés karstiques peuvent s'édifier à diverses échelles de temps, de l'infra-annuel au pluri-annuel (Perette 1999).

La méthode consiste à décrypter les éventuelles micropériodicités enregistrées dans le spéléothème laminé en effectuant une analyse spectrale sur la séquence continue la plus complète possible et de les identifier par analogie aux micropériodicités actuelles intervenant dans la dynamique climatique régionale ou supra régionale.

\section{L'AQueduC de FréJus : HYDROLOGIE ET FONCTIONNEMENT}

D'une longueur supérieure à $40 \mathrm{~km}$, l'aqueduc antique était alimenté essentiellement par la source de la Siagnole et par la source de la Foux (Fig. 1).

La source de la Siagnole (altitude $515 \mathrm{~m}$ ) est l'émissaire d'un bassin-versant d'altitude subalpin (plateau de Canjuers, altitude moyenne $1050 \mathrm{~m}$ ) soumis cependant à un régime méditerranéen franc (sécheresse estivale). Cette source qui alimente encore aujourd'hui plusieurs villages et bourgs du haut-Var a fait l'objet d'un suivi hydrologique du Service Régional d'Aménagement des Eaux (SRAE), en particulier

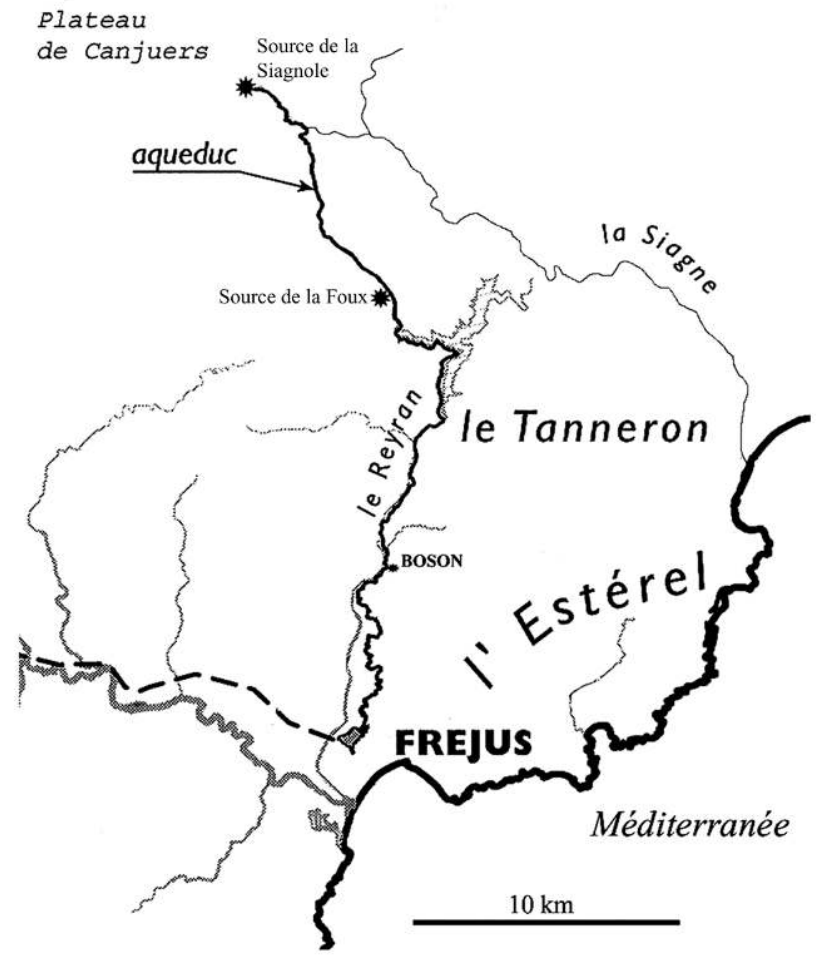

Figure 1 : Situation géographique et tracé de l'aqueduc antique de Fréjus.

Figure 1: Location and layout of the ancient aqueduct of Frejus. 
de septembre 1980 à décembre 1992. Le débit de la Siagnole est assez régulier et constant, même en été si la recharge hydrique a été bonne en hiver. Le déficit est surtout marqué en automne. Le diagramme biennal (1984-1985) de la figure 2 est sur ce point parfaitement représentatif du débit de la source.

La source de La Foux, située dans l'avant-pays provençal (altitude $151 \mathrm{~m}$ ), a un régime typiquement méditerranéen avec un fort étiage d'été.

En somme les deux sources, sans avoir un fonctionnement totalement identique, sont caratérisées par un débit étalé sur l'année entière avec un creux d'alimentation en été-automne. C’est un régime quasi-général dans le Midi méditerranéen en rapport avec les caractéristiques du climat, pérennes au moins depuis le milieu de l'Holocène (Petit-Maire et al., 1999). C'est ce régime que nous supposons avoir régi le concrétionnement de l'aqueduc, tout particulièrement sur le site de Boson (Fig. 1) où nous avons mené la présente étude. Un tel régime est tout à fait favorable à un type d'accrétion annuelle simple binaire lamine claire/ lamine sombre.

\section{LE CONCRÉTIONNEMENT SUR LE SITE DE Boson; ANNUITÉ DU DOUBLET}

Le concrétionnement calcitique est important en tout point de l'aqueduc de l'amont à l'aval. Localement il peut pratiquement obstruer tout le canal. Sur le site de Boson, le concrétionnement est bien laminé (laminite) et son épaisseur

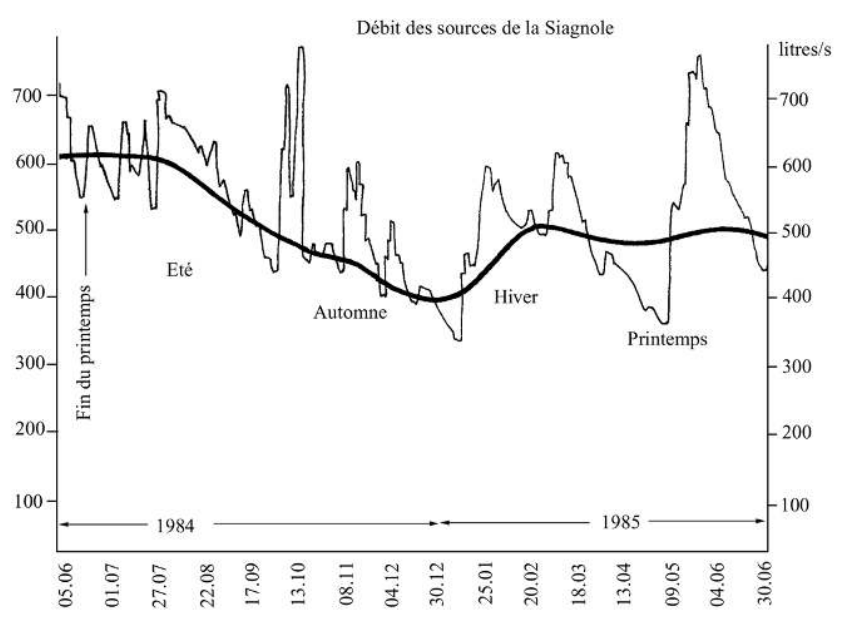

Figure 2 : Débits hydriques de la source de la Siagnole pour la période 1984-1985; données enregistrées (SRAE) et courbe lissée (lissage polynomial).

Figure 2: Hydrology of the Siagnole source for the 1984-1985 period; raw data (SRAE survey) and smoothed curve. atteint $20 \mathrm{~cm}$. Sa base repose sur le béton du canal et aucun grattage n'a perturbé sa croissance : il correspond manifestement à la première alimentation continue de l'aqueduc (Guendon et al., 2002). Si la mise en eau du canal se fait vers le milieu du $\mathrm{I}^{\text {er }}$ siècle apr. J.-C. (Gébara et al., 2002), le concrétionnement débuterait donc vers cette date.

Nous avons carotté avec un trépan le concrétionnement sur la paroi latérale gauche du canal à un endroit où il a $17 \mathrm{~cm}$ d'épaisseur. Il a été retaillé et monté en lame transparente (Fig. 3). Deux lames minces de contrôle minéralogique de la lamination confirment pleinement des observations microscopiques antérieures (Guendon et al., 2002). Les lamines claires sont constituées de grands cristaux orthomorphiques (sparite palissadique) de calcite translucide tandis que chaque lamine sombre est constituée d'une ou plusieurs couches de sparite oblongue séparées par un ou plusieurs microdiasthèmes à oxydes ferriques (Fig. 4) donnant une certaine opacité à la lamine. L'interprétation de la cristallisation, prenant comme base le régime des sources d'alimentation (Foux et Siagnole) et en admettant que ce régime n'a pas beaucoup changé depuis l'Antiquité, nous conduit à considérer que la lamine claire correspond à la période de hautes eaux (hiver+printemps+début de l'été), la lamine sombre serait le témoin de la période d'étiage (fin été et automne). Le fait est aussi montré par l'analyse des isotopes de l'oxygène des deux types de lamines (Bonté, 1999) : lamines sombres à $\delta{ }^{18} \mathrm{O}$ élevé témoins de paléotempératures estivales, lamines claires à $\delta{ }^{18} \mathrm{O}$ plus faible formées en période fraîche. C'est avec cette hypothèse d'un doublet annuel que le comptage et la mesure des lamines ont été réalisés. Dans le cas où cette hypothèse serait erronée, l'analyse spectrale ne fournirait aucun résultat cohérent qui pourrait montrer des composantes périodiques du climat connues.

\section{L'ANALYSE SPECTRALE}

\subsection{Méthode d'analyse des séries stratigraphiques}

Il existe plusieurs méthodes d'analyse spectrale permettant de rechercher et de quantifier les cyclicités des séries sédimentaires. Dans le logiciel Analyseries version 1.2 (Paillard et al., 1996) que nous avons utilisé, deux de ces méthodes sont particulièrement développées :

- le modèle de Fourier avec la méthode Blackman Tukey (1958) qui utilise une "transformée " permettant d'isoler les composants réguliers de la série temporelle sous forme d'ondes sinus et cosinus. Le résultat graphique de cette méthode est un spectre des fréquences à chacune desquelles 


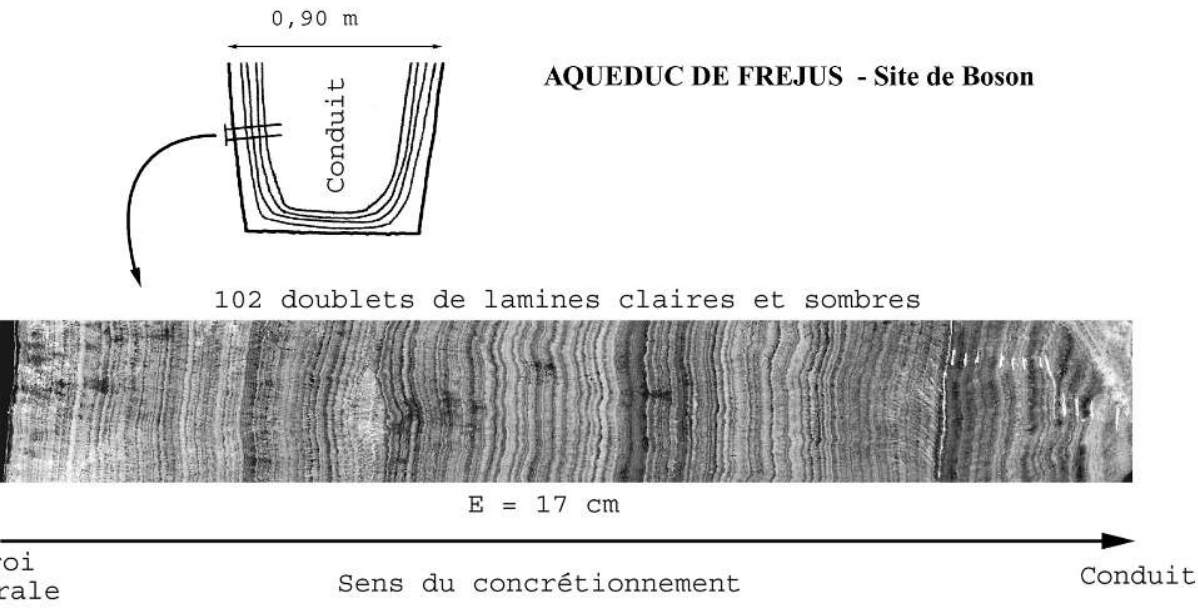

Figure 3 : Site de Boson; section du conduit et concrétionnement travertineux étudié. Figure 3: Boson site; section of the studied laminated travertine.

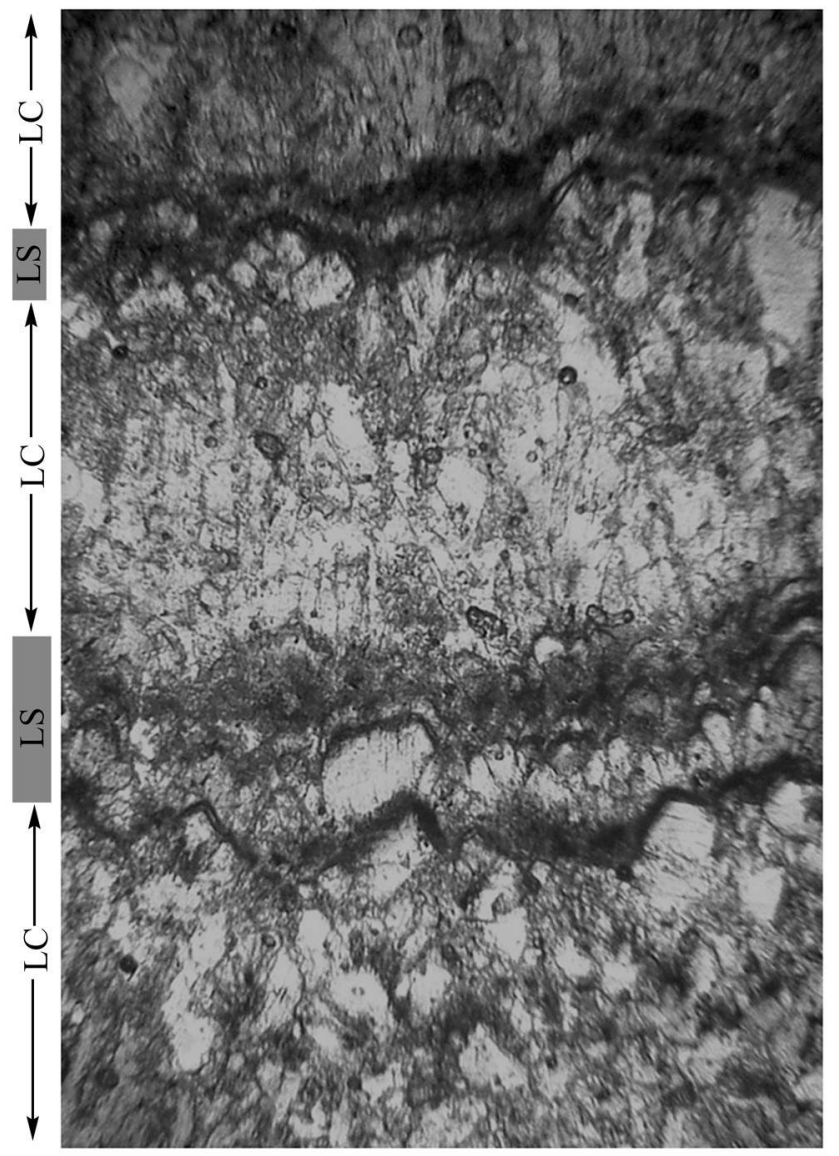

LC: lamine claire

Figure 4 : Site de Boson. Microstructure et agencement des deux types de lamines; hauteur de la section : $4 \mathrm{~mm}$.

Figure 4: Boson site; Microstuctures and arrangement of the two kinds of laminae; section height: $4 \mathrm{~mm}$.

ArcheoSciences, revue d'archéométrie, 30, 2006, p. 163-171

correspond un pic. L'amplitude du pic reflète directement la contribution relative de la fréquence considérée dans le signal initial.

- le modèle ARMA avec la méthode du maximum d'entropie (Ulrich et Bishop, 1975) correspond à la combinaison d'un processus autorégressif (AR) et d'un processus basé sur la moyenne mobile (MA).

La première méthode offre une bonne fiabilité pour des séries de données longues, la seconde permet d'augmenter la résolution.

Ces méthodes peuvent être complétées par une série d'opérations annexes de filtrage linéaire en utilisant en particulier le filtrage gaussien qui a pour effet de régulariser les pics selon une loi de distribution normale.

\subsection{Acquisition et traitement des données}

L'accrétion calcitique est considérée comme représentative de la saison humide et proportionnelle à sa durée. Lépaisseur du doublet serait, elle, un paramètre approchant de la pluviométrie annuelle. C'est ce dernier paramètre que nous cherchons à restituer sur la séquence temporelle étudiée et il convient alors de mesurer l'épaisseur des doublets.

Le comptage et la mesure des lamines ont été réalisés à la loupe binoculaire en utilisant un réglet micrométrique et en se basant sur les critères minéralogiques indiqués cidessus. Peu de cas ont posé des problèmes de séparation, ce qui nous a conduit à obtenir rapidement une série de 102 doublets (204 lamines). Leur épaisseur varie de 0,8 à $4 \mathrm{~mm}$ (Fig. 5, A). 
A - DONnees bRUtes
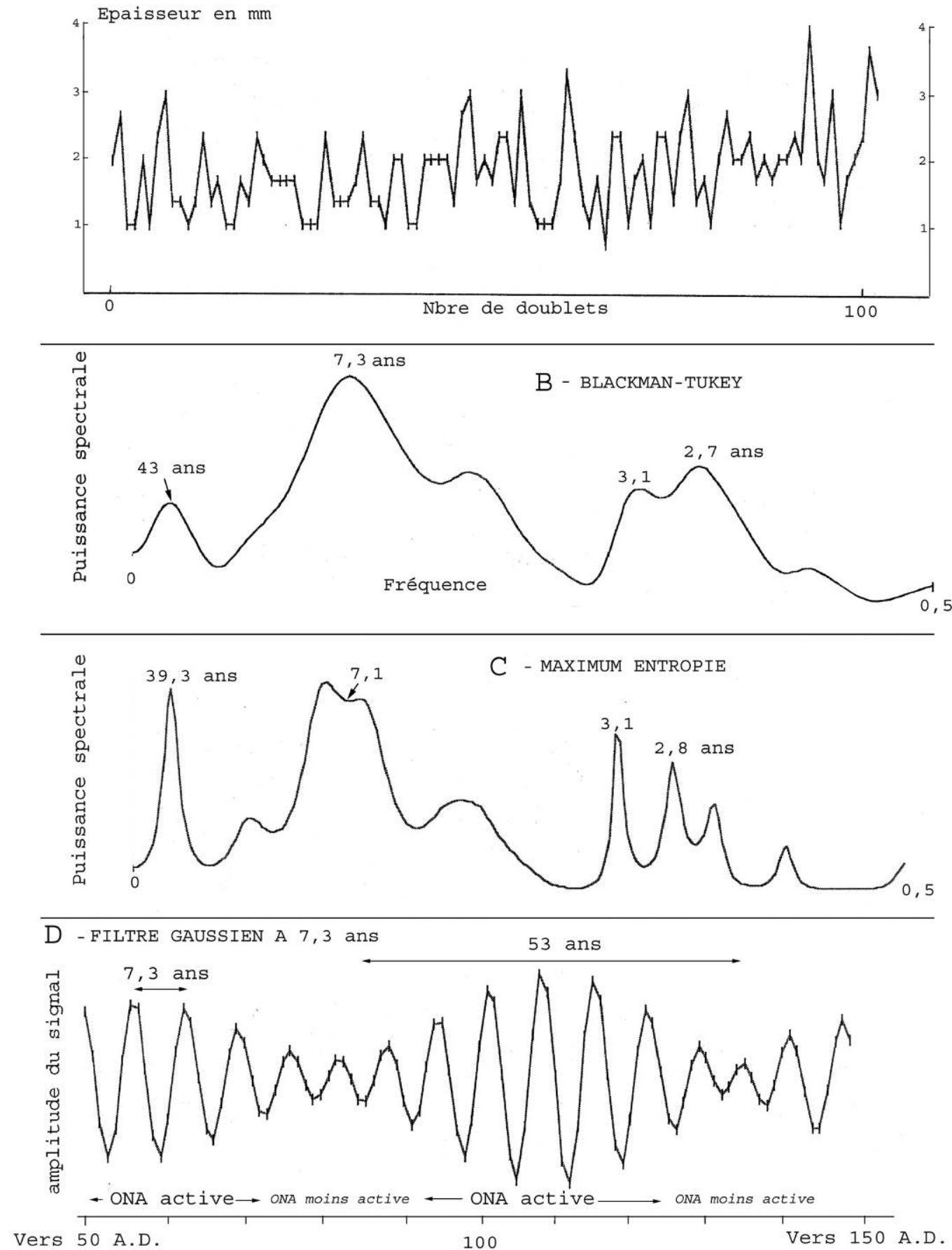

Figure 5 : Analyse spectrale de la série de l'épaisseur des 102 doublets du site de Boson, A. Données brutes; B. Méthode de BlackmanTuckey; puissance spectrale dans le domaine des fréquences (préparation et paramétrages dans le texte); C. Méthode du maximum d'entropie; puissance spectrale dans le domaine des fréquences (préparation o et paramétrages dans le texte) D. Filtrage gaussien à 7,3 ans; restitution du signal temporel et son interprétation en termes de climat par référence à l'ONA.

Figure 5: Spectral Analysis of the 102 doublets thickness series of Boson site, A. Raw data (thickness of the doublets); B. Blackman-Tuckey Method; Power spectrum in the frequency domain (preparation and parameter settings in the text); C. Maximum Entropy Method; Power spectrum in the frequency domain. (preparation and parameter settings in the text); D. Gaussian filtering at 7.3 years. Reconstructed time signal and its interpretation in terms of climate refering to the NAO. 
Un examen de la série brute (Fig. 5, A) montre une légère tendance à l'accroissement des valeurs. Une des conditions préalables à l'application des méthodes d'analyse spectrale est de supprimer la tendance afin de rendre la série «stationnaire». Ceci est fait optionnellement sur le logiciel Analyseries par un lissage qui opère par soustraction d'une régression linéaire d'ajustement des données au moindre carré. De plus, afin de réduire le "bruit " de la série brute, l'application des deux méthodes spectrales est réalisée à la demande non pas sur les valeurs brutes mais sur la fonction d'autocorrélation de la série étudiée qui conserve tous les composants fréquentiels du signal.

D’une manière plus générale, avec ce logiciel, la préparation de l'analyse se fait en introduisant un paramétrage (en particulier un rapport confiance/résolution) dont l'opportunité apparaît en testant plusieurs valeurs (Paillard et al., 1996).

\subsection{Résultats et première approche climatique}

1/ Blackman-Tuckey (Fig. 5, B)

En recherchant une résolution moyenne et une fiabilité moyenne (lissage modéré des pics), le spectre de fréquence fait apparaître un fort pic à 7,3 années et des pics plus discrets mais assez puissants à 43 ans pour les plus hautes fréquences et deux pics à très haute fréquence à 3,2 et 2,7 années.

\section{2/ Maximum entropie (Fig. 5, C)}

En travaillant à résolution moyenne, le spectre des fréquences fait apparaître les mêmes groupements de pics que la transformée de Fourier B-T : un pic à la fréquence de 39,3 années, un pic (un peu dédoublé) à la fréquence de 7,1 années et un paquet des trois pics à 3,1, 2,85 et 2,5 ans.

Ces deux résultats se confortent : ils laissent transparaître l'existence de deux groupes de pics énergétiques, l'un à haute (période de 7 ans) et très haute (vers 3 ans) fréquence, l'autre à basse fréquence (vers 40 ans). La réalité de cette dernière, malgré la brièveté de la séquence (102 mesures) est attestée en faisant un filtrage gaussien de la série brute conservant uniquement la fréquence principale à 7 ans (Fig. 5, D). Le signal obtenu laisse apparaittre une longue modulation de près de 50 ans sur la courbe, qui est assimilable, compte tenu du degré de précision de la mesure, à celle de 43 ans révélée par l'analyse spectrale.

Cette bipartition en deux groupes (basse d'un côté, haute et très haute de l'autre) des récurrences climatiques rappelle fortement l'Oscillation Nord-Atlantique (Hurrel et Van Loon, 1997).
En corollaire de la cohérence du résultat de l'analyse spectrale et de l'identification du signal de l'ONA dans l'enregistrement travertineux de Boson, l'hypothèse de l'annuité du doublet que nous avons faite est pleinement validée. La chronologie dudit concrétionnement est donc de 50 A.D. à 150 A.D. environ.

\section{ESSAI D'INTERPRÉTATION CLIMATIQUE}

L'Oscillation Nord Atlantique (ONA), liée à la différence de pression entre l'anticyclone des Açores et la dépression d'Islande, a un rôle déterminant dans l'Atlantique Nord et en Europe occidentale. En France méridionale et dans les Alpes françaises son rôle dans la période historique a été montré à plusieurs reprises (Perrette, 1999; Chapron et al., 2002; Dubar in Foucras et al., 2005). À l'échelle de quelques décennies, l'ONA oscille entre deux modes : un mode actif avec indice positif caractérisé par un régime de fort vent d'ouest, pluviosité forte et température douce et un mode passif avec indice négatif au cours duquel le front nordique est prépondérant et amène des conditions de froid et de sécheresse.

Les études sur l'ONA (Appenzeller et al., 1998) tendent à montrer que lors des phases actives, les périodicités sont dans les hautes fréquences (5-15 ans) alors qu'en phase passive c'est uniquement la basse fréquence qui apparaît (40-50 ans). La périodicité de 7-8 ans (ici 7,3 ans) en particulier a fait l'objet d'une attention toute particulière des météorologistes quant à ses liens avec l'ONA (Da Costa et Colin, 2002).

Bien sûr il existe des phases transitoires au cours desquelles les deux types de fréquences se manifestent. On peut dire alors que le climat n'est pas strictement déterminé par rapport à l'ONA. C'est le cas de l'enregistrement de Boson où cependant la haute fréquence (ONA +) est dominante.

Pour améliorer l'interprétation et compte tenu de la robustesse du pic à 7,3 ans, un filtrage gaussien à cette fréquence dominante a été réalisé sur la durée du concrétionnement (Fig. 5, D). Une modulation secondaire en intensité apparaît, dont la période mesurée est un peu supérieure à 50 ans. C'est bien la "basse fréquence » détectée par l'analyse spectrale à quelques années près. Il y a donc bien une interférence des deux types de fréquence : sur la durée en question on peut dire que des périodes de quelques décennies à ONA active alternent avec des périodes à ONA moins active. En terme de climat, cela peut se résumer (Fig. 6) comme une période d'ambiance générale plutôt tempérée-humide avec des phases d'humidité et de température plus marquées alternant avec des phases plus froides et plus sèches. Il est 


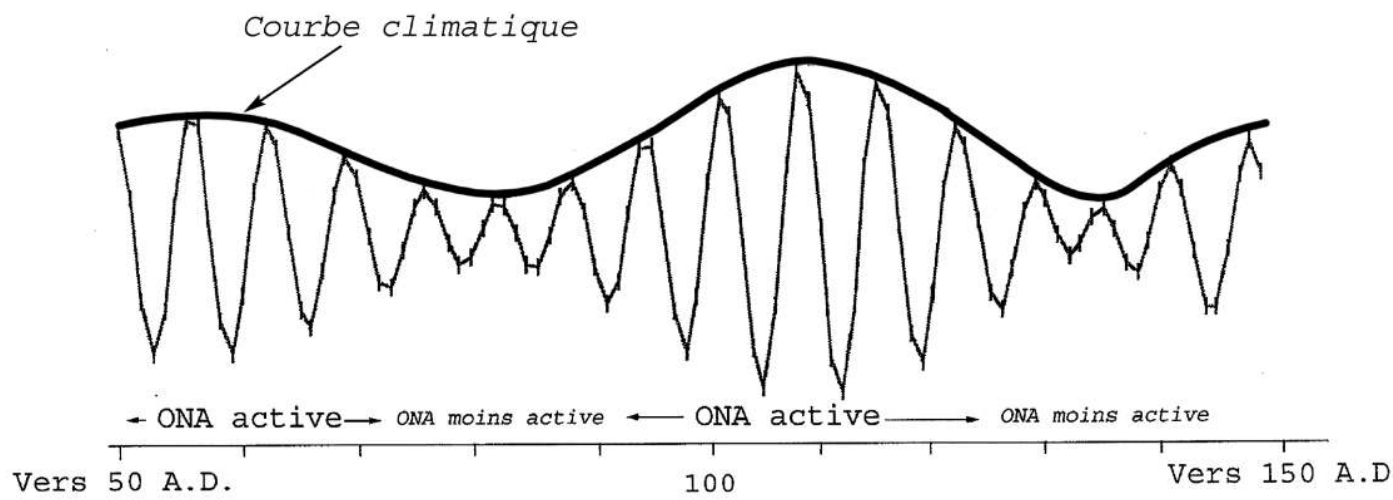

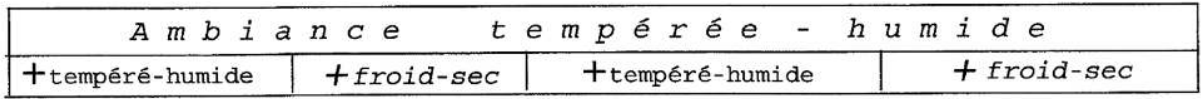

Figure 6 : Courbe climatique (50-150 apr. J.-C.) obtenue à Boson à partir de la série filtrée à 7,3 ans (fig. 5-D) et son interprétation.

Figure 6: Climatic curve from obtained in Boson site statrting from the filtered at 7,3 years series and its interpretation.

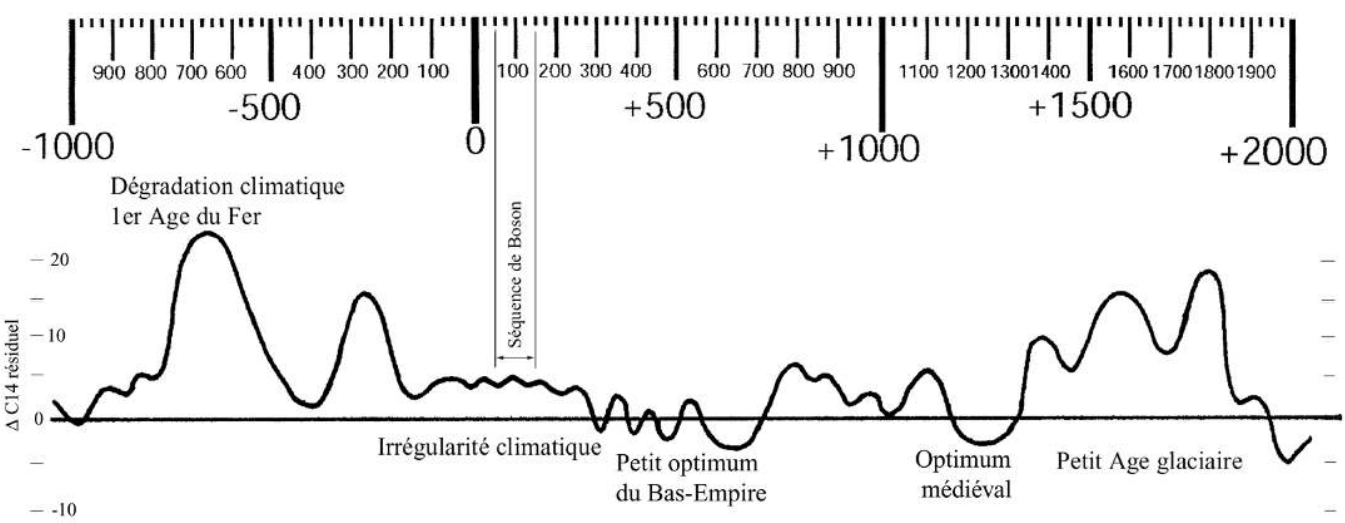

Figure 7 : La séquence climatique de Boson replacée sur la courbe simplifiée du $14 \mathrm{CO}_{2}$ de Stuiver et Braziunas (1993) avec indication des phases de Berger et al. (2003).

Figure 7: The climatic Boson sequence replaced on the simplified curve of $14 \mathrm{CO}_{2}$ of Stuiver and Braziunas (1993) showing the phasis of Berger et al. (1993).

possible de plus de tracer une courbe climatique synthétique (température plus humidité) qui cependant n'est pas strictement calée chronologiquement (Fig. 6). Pour une meilleure compréhension de cette courbe locale nous l'avons replacée sur la courbe du delta C14 de Stuiver et Braziunas, 1993 (Fig. 7). Cette courbe " globale » représente en théorie l'activité solaire et donc indirectement les variations du climat de l'Holocène. La séquence de Boson, conformément au caractère que lui attribue l'interprétation de l'analyse spectrale, correspond à une période de calme climatique relatif.

\section{DISCUSSION, PERSPECTIVE DE RECHERCHE}

Plusieurs reconstitutions du climat de l'époque romaine dans le Midi méditerranéen ont été proposées ces dernières années à partir de données paléoenvironnementales locales ou régionales (Jorda et Provansal, 1996; Berger et al., 2003; Arnaud-Fassetta et al., 2003; Van Der Leeuw et al., 2005). Ces travaux font appel largement à la paléohydrologie, parfois au couvert végétal et aux paléosols de cette époque. Il est ainsi montré que l'époque romaine prend place dans une période de calme érosif entre les crises du premier Âge du 
Fer (viII ${ }^{\mathrm{e}} \mathrm{V}^{\mathrm{e}}$ siècle av. J.-C.) et le Petit Âge Glaciaire ( $\mathrm{xv}^{\mathrm{e}-}$ $\mathrm{XIX}^{\mathrm{e}}$ siècle apr. J.-C.). Pour les auteurs cités, la période qui nous concerne, le Haut-Empire, serait une période de transition et d'irrégularité climatique accentuée par l'action de l'homme et conduisant à un "optimum» du Bas-Empire. En somme cette période n'aurait pas de caractère franchement marqué sur le plan du climat. Les arguments restent fragiles car uniquement déduits du paléoenvironnement qui est bien sûr sous la double influence de l'homme et du climat. L'analyse spectrale, elle, permet d'identifier des composantes physiques périodiques, reconnues comme liées au climat. On valide ainsi les données environnementales de paléohydrologie. Dans le cas présent, notre résultat conforte cette perception de période à caractère peu tranché. Il reste à étayer ce résultat isolé par d'autres analyses de ce type pour obtenir une série chronologique plus longue permettant de connaître la variabilité au moins sur deux à trois siècles.

\section{Bibliographie}

Appenzeller, C., Stocker, T. F. et Anklin, M., 1998. North Atlantic Oscillation dynamics recorded in Greenland Ice Cores, Science, 282, p. 446-449.

Arnaud-Fassetta, G. et Landure, C., 2003. Hydroclimatic hazards, vulnerability of societies and fluvial risk in the Rhône delta (Mediteerranean France) from the Greek period to the Early Middle Ages in E. Fouache (ed.), The Mediterranean World Environment and History, Elsevier, 2003, p. 51-76.

BAKER, A. et Genty, D., 2003. Comment on a "Test of annual resolution in stalagmites using tree rings", Quaternary Research, 59, p. 476-478.

Berger, J.-F., Fiches, J.-L. et Gazenbeek, M., 2003. Origin of fluvial fluctuations of the River Vidourle and their effect on the organisation ans evolution of the site of Ambrussum (Hérault, Fr.) between the Iron Age and lat Antiquity, in E. Fouache (éd.), The Mediterranean World Environment and History. Elsevier, 2003, p. 77-108.

Berthelot M., Genin A. et Gourdon D., 2004. Variabilité interannuelle des précipitations dans les Alpes du Sud pour la période 1951/2000 : analyses statistiques des séries. [http:// perso.wanadoo.frlalexandre. letort/berthelolalpessud.htm]

Blackman, R. B. et TuCKey, J. W., 1958. The measurement of power spectra from the point of view of communication engineering, New York, Dovar Publication Inc., 190 p.

BoNTÉ, S., 1999. Essai d'interprétation du fonctionnement de l'aqueduc romain de Fréjus à partir de l'étude pétrographique et isotopique des concrétions internes des conduits, DEA « Environnement et Archéologie », Paris, 57 p.
Burns, S. J., Fleitmann, D., Mudelsee, M., NefF, U., Matter, A. et ManginI, A., 2002. A 780-year annually resolved record of India Ocean monsoon precipitation from a speleothem from south Oman, Journal of Geophysical Research, 107, p. 4434-4441.

Chapron, E., Desmet, M., De Putter, T., Loutre, M.-F, Beck, C. et Deconninck, J.-F., 2002. Climatic variability in the northwestern Alps, France, a evidenced by 600 years of terrigenous sedimentation in Lake Le Bourget, The Holocene, 12, 2, p. 177-185.

Da Costa, E. D. et Colin de Verdière, A., 2002. The 7.6 year North Atllantic Oscillation. Quaterly Journal of Research of the Meteorological Society, 128, p. 797-817.

Foucras, J., Garzcinsky, P. et Dubar, M. (coll.), 2005. L'aqueduc romain des Bouillides, Gallia, 62, p. 13-34.

Gébara, C., Michel, J.-M. et Guendon, J.-L., 2002. L'aqueduc romain de Fréjus, sa description, son histoire et son environnement, supplément $\mathrm{n}^{\circ} 3$ de la Revue Archéologique de Narbonnaise, 320 p.

Genty, D. et QuinIf, Y., 1996. Annually laminated sequences in the internal structure of some Belgian stalagmites - importance for paleoclimatology, Journal of Sedimentary Research, 66,1, p. $275-288$.

Guendon, J.-L., Huon, S., Parron, C. et Bonté, S., 2002. Les concrétions calcaires témoins du fonctionnement de l'Aqueduc in Gebara et al. (dir.), L'aqueduc romain de Fréjus, sa description, son histoire et son environnement, supplément $\mathrm{n}^{\circ} 3$ de la Revue Archéologique de Narbonnaise, p. 163-215.

Hurrel, J. W. et Van Loon, H., 1997. Decadal variations in climate associated with the North Atlantic Oscillation. Climatic Change, 36, p. 301-326.

Jorda, M. et Provansal, M., 1996. Impact de l'anthropisation et du climat sur le détritisme en France du sud-est (Alpes du sud et Provence), Bulletin de la Société Géologique de France, 167-1, p. 159-169.

Paillard, D., Labeyrie, L. et Yiou, P., 1996. Macintosh program performs time-series analysis, Eos Transactions $A G U, 77$, p. 379.

Perrette, Y., 1999. Les stalagmites : archives environnementales et climatiques à haute résolution; présentation des protocoles d'étude et premiers résultats sur des spéléothèmes du Vercors, Karstologia, 34, p. 23-44.

Petit-Maire, N. (coord.), Antoine, P., Beaulieu, J.-L., Bintz, P., Brugal, J.-Ph., Girard, M., Morzadec, M.-T., RenaultMiskovsky, J., Roblin, A.-M. et Van Vliet-Lanoé, B., 1999. La France à l'optimum climatique holocène, 8000+/-1 000 ans B.P. Carte au 1/1000 000, Publication ANDRA-CNF/ INQUA, Paris.

Plaut G., Ghil M. et Vautard R., 1995. Interrannual and interdecadal variability in 335 years of Central England temperature, Science, 268, p. 710-713. 
Stuiver, M. et Braziunas, T., 1993. Ocean, climate and atmospheric ${ }^{14} \mathrm{CO}_{2}$ : an evaluation of causal and spectral relationship, The Holocene 3, p. 289-305.

Ulrich, T. J. et BISHOP, T. N., 1975. Maximum entropy spectral analysis and autoregressive decomposition . Rev. Geophys. Space Phys., 13, p. 183-200.
Van Der Leuw, S. E., the Archeomedes research team, 2004. Climate, hydrology, land use and environmental degradation in the lower Rhône Valley during Roman period, Compte Rendus Geosciences, 337, p. 9-27.

WANNER, H, 1999. Le balancier de l'Atlantique nord, La recherche, 321, p. 72-73. 